\title{
Feasibility Study for Spectroscopy of Pionic Atoms with Unstable Nuclei
}

\author{
Y.N. Watanabe ${ }^{*}{ }^{a}$ H. Fujioka, ${ }^{b}$ R.S. Hayano,${ }^{a}$ K. Itahashi, ${ }^{c}$ M. Niikura, ${ }^{a}$ T. Nishi, ${ }^{a}$ \\ Y.K. Tanaka ${ }^{a}$ \\ ${ }^{a}$ Department of Physics, University of Tokyo, Tokyo, JAPAN \\ ${ }^{b}$ Department of Physics, Kyoto University, Kyoto, JAPAN \\ ${ }^{c}$ Nishina Center, RIKEN, Saitama, JAPAN \\ E-mail: ywatanabe@nucl.phys.s.u-tokyo.ac.jp
}

\begin{abstract}
We are preparing for missing-mass spectroscopy of deeply bound pionic atoms with unstable nuclei. We make use of inverse kinematics of $\left(d,{ }^{3} \mathrm{He}\right)$ reaction by employing RI beams and determine the missing-mass of the reaction by measurement of the energy and the angle of the ${ }^{3} \mathrm{He}$. The feasibility of the spectroscopy was studied based on a setup with a pure deuterium active target with respect to the missing-mass resolution and the yield of the pionic atoms. We concluded that the missing-mass resolution is about $500 \mathrm{keV}$ (FWHM) and the yield of the pionic $1 s$ state is 20 counts/day.
\end{abstract}

XV International Conference on Hadron Spectroscopy-Hadron 2013

4-8 November 2013

Nara, Japan

${ }^{*}$ Speaker. 


\section{Introduction}

Spectroscopy of pionic atoms has been providing precision information on the pion-nucleus strong interaction. Among many of pionic atoms, deeply bound states such as $1 s$ states in relatively heavy nuclei, have notable sensitivity of the $s$-wave interaction due to the large overlap between the pion distribution and the nuclear density distribution. Phenomenologically, pion-nucleus interaction is well described by an optical potential with several complex parameters.

There is a parameter called isovector scattering length $b_{1}$ that parametrizes the potential depth proportional to the density difference between the neutron and the proton distributions. The $b_{1}$ is known to play a very important role in studying the chiral symmetry breaking and its partial restoration in nuclear medium. The order parameter of chiral symmetry breaking $|\langle\overline{\mathrm{q}} \mathrm{q}\rangle|$ is related to the isovector scattering length $b_{1}[1,2,3,4]$. Thus, precision determination of $b_{1}$ may provide quantitative information on the magnitude of the partial restoration of chiral symmetry at the normal nuclear density $\rho_{0}$.

Precisely speaking, deduced knowledge of in-medium $b_{1}$ from the spectroscopy of pionic atoms is only at $\sim 0.6 \rho_{0}$. This situation can be seen clearly when we plot the overlap between pion distribution and the nuclear density. The overlap shows a steep rise at the density $\sim 0.6 \rho_{0}$ regardless of the nucleus in which the pion is captured. This picture is understood in the structure of the pionic atoms in a balance between the repulsive strong interaction and the attractive Coulomb interaction.

In past experiments for the spectroscopy of deeply bound pionic atoms, missing-mass of the reactions ${ }_{Z}^{A} \mathrm{HI}\left(d,{ }^{3} \mathrm{He}\right) \pi^{-} \otimes_{Z}^{A-1} \mathrm{HI}$ were measured, where ${ }_{Z}^{A} \mathrm{HI}$ denotes a heavy ion and $\pi^{-} \otimes_{Z}^{A-1} \mathrm{HI}$ a pionic atom. Deeply bound pionic states of isotopes, ${ }^{115,119,123} \mathrm{Sn}$ and ${ }^{205,207} \mathrm{~Pb}$, were measured and the $b_{1}$ parameter was deduced $[5,6,7,8,9]$. The achieved results showed reduction of the $|\langle\overline{\mathrm{q}} \mathrm{q}\rangle|$ at $\rho_{0}$ by about $35 \%$, experimentally indicating partial restoration of chiral symmetry at a high density medium for the first time. Currently, a series of new experiments has been in preparation to obtain a better precision at RI Beam Factory, RIKEN.

As a next step of the spectroscopy of pionic atoms, we plan to perform a missing-mass spectroscopy of pionic atoms with unstable nuclei, which develops potential to investigate density dependence of the $|\langle\overline{\mathrm{q}} \mathrm{q}\rangle|$. As mentioned, deduced knowledge of in-medium $b_{1}$ is limited to the density $\sim 0.6 \rho_{0}$. Pionic atoms with unstable neutron-rich nuclei may provide opportunity to break the limitations and approach investigations of $|\langle\bar{q} \mathrm{q}\rangle|$ at smaller densities because the pion is pushed outward by the repulsive interaction with the valence neutron and we may gain information of $|\langle\overline{\mathrm{q}} \mathrm{q}\rangle|$ at lower densities.

\section{Experimental Principles and Experimental Setup}

Spectroscopy of pionic atoms with unstable nuclei is not a simple continuation of the present experiments. We need to employ inverse kinematics reaction, $d\left(\mathrm{HI},{ }^{3} \mathrm{He}\right) \pi^{-} \otimes_{Z}^{A-1} \mathrm{HI}$ simply because the unstable nuclei cannot be used as a fixed target. Figure 1 presents a schematic drawing of the reaction under the recoil-free kinematical condition in the center of mass frame, in which the momentum transfer of the reaction is zero and pionic $1 s$ state cross section is enhanced. The kinematical condition requires the incident beam energy to be $250 \mathrm{MeV} /$ nucleon. We will adopt 
missing-mass measurement to perform the $Q$-value spectroscopy. The reaction $Q$-value is deduced by measurement of the ${ }^{3} \mathrm{He}$ energy and the angle. Note that the energy uncertainty of the incident beam does not have major contribution to the $Q$-value resolution.

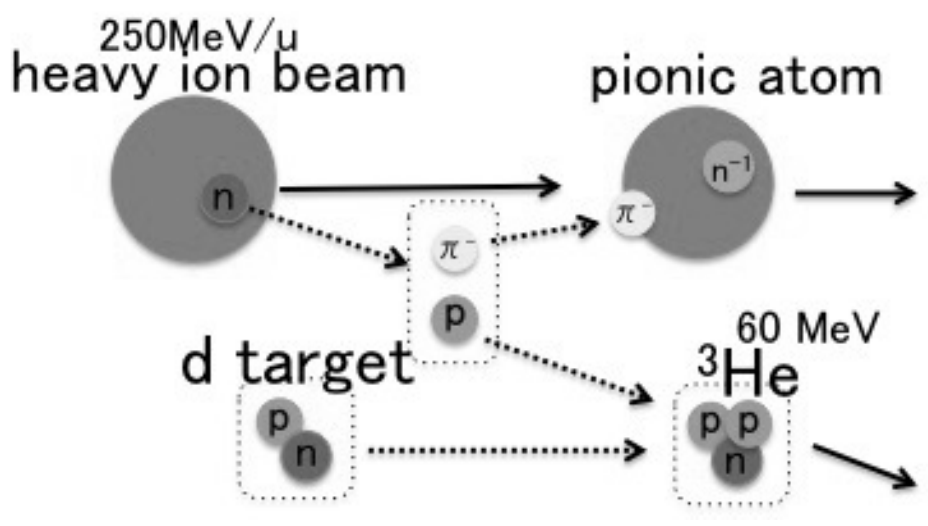

Figure 1: Schematic drawing of $d\left(\mathrm{HI},{ }^{3} \mathrm{He}\right)$ reaction. A heavy ion beam is injected into a deuteron target and a pionic atom and a ${ }^{3} \mathrm{He}$ are produced. Under the recoilless condition in the $\mathrm{CM}$ frame, the energy of the incident heavy ion is $250 \mathrm{MeV} / \mathrm{u}$ and energy of the recoil ${ }^{3} \mathrm{He}$ is about $60 \mathrm{MeV}$.

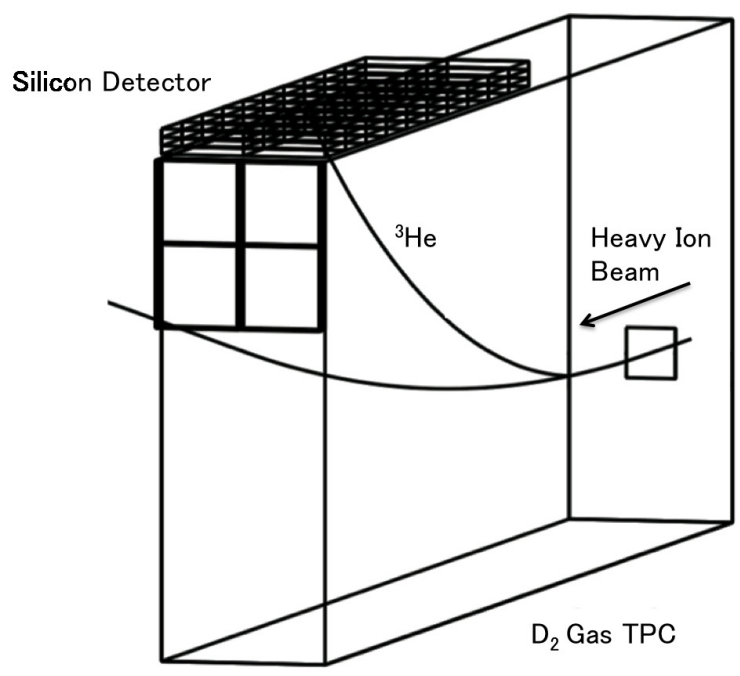

Figure 2: Conceptual design of the experimental setup with the AT-TPC and the silicon detectors. A magnetic field is applied in the perpendicular direction to the beam and parallel with the electron drift direction. A heavy ion beam is injected into the $1 \mathrm{~m}$-long AT-TPC and the ${ }^{3} \mathrm{He}$ track and energy loss are measured in the AT-TPC and the full energy is measured by the silicon detectors.

One of the difficulties of the experiment in view of the $Q$-value resolution resides in the fact that the emitted ${ }^{3} \mathrm{He}$ has a considerably small energy of $\sim 60 \mathrm{MeV}$. We need to determine the ${ }^{3} \mathrm{He}$ energy at a precision of $\sim 60 \mathrm{keV}$ and the emission angle of $\sim 5 \mathrm{mrad}$. 
Taking into account the energy loss of the ${ }^{3} \mathrm{He}$ in various materials, we came to consider a pure deuterium gas active target as the core component of the experimental setup.

From here on, we will consider an experimental setup using an active target time projection chamber (AT-TPC), and evaluate the experimental feasibility mainly in terms of the spectroscopic resolution and the yield. A schematic view of the experimental setup is shown in Fig. 2. An AT-TPC filled with deuterium gas is placed in a uniform magnetic field of about 1 Tesla.

A $250 \mathrm{MeV} / \mathrm{u}$ heavy ion beam is impinged on the AT-TPC in order to induce the $d\left(\mathrm{HI},{ }^{3} \mathrm{He}\right)$ reaction. The ${ }^{3} \mathrm{He}$ is emitted in the forward direction and separated from the incident beam by the magnetic field due to a large difference in the magnetic rigidity of ${ }^{3} \mathrm{He}(1 \mathrm{Tm})$ and the beam $(7 \mathrm{Tm})$. The ${ }^{3} \mathrm{He}$ track is measured by the TPC and the full energy by the $2000 \mu \mathrm{m}$-thick silicon detectors located inside the TPC gas volume.

Table 1: Simulation conditions.

\begin{tabular}{c|c}
\hline${ }^{132} \mathrm{Sn}$ beam intensity & $10^{6} / \mathrm{s}$ \\
\hline $\mathrm{D}_{2}$ gas thickness & $1 \mathrm{~m}$ \\
\hline Cross section & $5 \times 10^{-2} \mu \mathrm{b}$ \\
\hline $\begin{array}{c}\text { AT-TPC position resolution }(\sigma) \\
\text { Energy resolution }(\sigma) \text { of } \mathrm{Si} \\
\text { detector at } 60 \mathrm{MeV}^{3} \mathrm{He}\end{array}$ & $500 \mu \mathrm{m}$ \\
\hline
\end{tabular}

We estimated the $Q$-value resolution and the yield of the pionic $1 s$ state in ${ }^{131} \mathrm{Sn}$ nuclei by a simulation under the conditions listed in Table 1 . The current available intensity of ${ }^{132} \mathrm{Sn}$ beam is about $10^{5} / \mathrm{s}$ using $10 \mathrm{pnA}{ }^{238} \mathrm{U}$ beam in RIBF, but we expect the beam intensity of $10^{6} / \mathrm{s}$ will be usable in the future. The pionic atom formation cross section of the $d\left({ }^{132} \mathrm{Sn},{ }^{3} \mathrm{He}\right) \pi^{-} \otimes{ }^{131} \mathrm{Sn}$ reaction for the $1 s$ state was estimated based on a linear extrapolation from a series of experimental measurements of pionic Sn isotopes [9]. TPC position resolution and energy resolution of $\mathrm{Si}$ detector are assumed to be $500 \mu \mathrm{m}$ and $0.1 \%$ respectively.

Table 2: Contributing factors to the $Q$-value resolution.

\begin{tabular}{c|c}
\hline Resolution contribution & $\begin{array}{c}\text { Contribution to } \\
Q \text {-value resolution } \\
\text { (FWHM) [keV] }\end{array}$ \\
\hline \hline Energy straggling & 350 \\
\hline Vertex position resolution & 150 \\
\hline $\begin{array}{c}\text { Energy resolution of } \\
\text { Si detectors }\end{array}$ & 350 \\
\hline \hline Total & 500 \\
\hline
\end{tabular}

As listed in Table 2, the large contributions to the $Q$-value resolution are arising in the energy straggling of the low energy ${ }^{3} \mathrm{He}$ in the active target volume and the energy resolution of the silicon detectors. Vertex position resolution also contributes through estimation of the integrated energy loss along the track of the ${ }^{3} \mathrm{He}$. Based on the above assumed conditions, the $Q$-value resolution and 
the yield of the $1 s$ pionic atom formation is evaluated to be $500 \mathrm{keV}$ (FWHM) and 20 counts/day, respectively.

\section{Discussion and future perspective}

The estimated $Q$-value resolution of $500 \mathrm{keV}$ (FWHM) is good enough and is comparable with past experiments with normal kinematics $(\sim 400 \mathrm{keV})$. Such high resolution is essential in resolving the pionic $1 s$ state from the other states since the typical energy difference between the $1 s$ state and the other states is about $1 \mathrm{MeV}$.

The yield of pionic $1 s$ state of 20 counts/day is sufficient to observe the peak structures of pionic states with ten days measurement. The peak is located on a constant background of $\sim 4 \mu \mathrm{b} / \mathrm{str} / \mathrm{MeV}$ originating in nuclear excitation not associated with pion production.

We have not discussed possible major sources of background in the above measurement. Large sources are emerging from scattered deuterons by the incident $\mathrm{HI}$ beam and protons originating in the deuteron breakup. These particles will be identified and rejected by combining the information on the radius of the tracks, energy deposit in the AT-TPC, and the full energy deposit in the silicon detectors.

We are now testing a silicon detector using an $\alpha$ source and the estimated energy resolution is $\sim 0.3 \%(\sigma)$ at $5.5 \mathrm{MeV}^{4} \mathrm{He}$. We will further carry out the test to achieve a better resolution by reducing electric noise and cooling the detector. After the construction of detectors, we will carry out an experiment using stable nuclei beam in order to confirm the experimental feasibility taking into account the beam intensity, quality and availability.

This work is partly supported by the Grants-in-Aid for Scientific Research No. 24105712.

\section{References}

[1] M. Gell-Mann, R.J. Oakes, and B. Renner, Phys. Rev. 175, (1968) 2195.

[2] E. E. Kolomeitsev, N. Kaiser, and W. Weise, Phys. Rev. Lett. 90, (2003) 092501.

[3] D. Jido, T. Hatsuda, and T. Kunihiro, Phys. Lett. B 670, (2008) 109.

[4] N. Ikeno et al., Prog. Theor. Phys., 126, (2011) 483.

[5] H. Gilg et al., Phys. Rev. C 62, (2000) 025201.

[6] K. Itahashi et al., Phys. Rev. C 62, (2000) 025202.

[7] H. Geissel et al., Phys. Rev. Lett. 88, (2002) 122301.

[8] H. Geissel et al., Phys. Lett. B 549, (2002) 64.

[9] K. Suzuki et al., Phys. Rev. Lett. 92, (2004) 072302. 